Quim. Nova, Vol. 36, No. 1, 40-45, 2013

\title{
SÍNTESE E CARACTERIZAÇÃO DE HIDROGÉIS COMPÓSITOS A PARTIR DE COPOLÍMEROS ACRILAMIDA- ACRILATO E CAULIM: EFEITO DA CONSTITUIÇÃO DE DIFERENTES CAULINS DO NORDESTE BRASILEIRO
}

\author{
Carlos W. de Q. Brito e Francisco H. A. Rodrigues* \\ Coordenação de Química, Universidade Estadual Vale do Acaraú, Av. da Universidade, 850, Campus da Betânia, 62040-370 \\ Sobral - CE, Brasil \\ Marcus V. da S. Fernandes, Lindomar R. D. da Silva, Nágila M. P. S. Ricardo e Judith P. A. Feitosa \\ Departamento de Química, Universidade Federal do Ceará, Campus do Pici, 60455-760 Fortaleza - CE, Brasil \\ Edvani C. Muniz \\ Departamento de Química, Universidade Estadual de Maringá, Av. Colombo, 5790, 87020-900 Maringá - PR, Brasil
}

Recebido em 25/2/12; aceito em 27/7/12; publicado na web em 27/11/12

\begin{abstract}
SYNTHESIS AND CHARACTERIZATION OF POLY (ACRYLAMIDE-CO-ACRYLATE) AND KAOLIN HYDROGEL COMPOSITES: EFFECT OF THE CONSTITUTION OF DIFFERENT KAOLINS FROM NORTHEASTERN BRAZIL. Superabsorbent hydrogels based on poly (acrylamide-co-acrylate) and different kaolins, were prepared by free-radical aqueous copolymerization. FTIR and WAXS techniques were employed for characterization of a series of hydrogels, obtained by varying the percentage of clay, crosslinking and constitution of kaolin. The water absorbency at equilibrium $\left(\mathrm{W}_{\mathrm{eq}}\right)$ decreased with increasing clay content and the amount of crosslinking agent. Superabsorbent hydrogel $\left(\mathrm{W}_{\mathrm{eq}}>1084 \mathrm{~g} \mathrm{H}_{2} \mathrm{O} / \mathrm{g}\right.$ gel$)$ was obtained as $10 \mathrm{wt} \%$ of white kaolin and $0.05 \mathrm{~mol} \%$ of crosslinking agent were used. The hydrogel proved sensitive to $\mathrm{pH}$ variation and the presence of salts.
\end{abstract}

Keywords: superabsorbent hydrogels; clay; agriculture.

\section{INTRODUÇÃO}

Os hidrogéis podem ser definidos como materiais constituídos por redes poliméricas hidrofílicas química ou fisicamente reticuladas, capazes de absorver e reter grande quantidade de água e/ou fluídos biológicos sem perder sua forma tridimensional (3D). São utilizados na atividade agrícola como condicionadores de solo, principalmente por aumentarem a capacidade de armazenamento de água do solo. ${ }^{1,2}$ Um condicionador de solo é definido como todo material orgânico, sintético, natural ou quimicamente modificado que, adicionado ao solo, altera favoravelmente suas propriedades físicas e estruturais. ${ }^{3}$

O processo de intumescimento de um hidrogel é governado por fatores físicos intrínsecos à rede $3 \mathrm{D}$ e a fatores externos. Alguns fatores físicos, tais como, presença de grupos hidrofílicos, $\left(-\mathrm{OH},-\mathrm{NH}_{2}\right.$, $-\mathrm{COOH},-\mathrm{CONH}_{2},-\mathrm{SO}_{3} \mathrm{H}$ ) na estrutura da cadeia polimérica (lateralmente ou na cadeia principal), menor densidade de reticulação e uma alta flexibilidade da rede polimérica, contribuem positivamente para um maior intumescimento do material. ${ }^{4}$ Por outro lado, a presença de grupos hidrofóbicos, maior densidade de reticulação e a presença de íons desfavorecem a absorção de água pelo gel. Mudanças de $\mathrm{pH}$, força iônica, temperatura, composição de solvente são alguns dos fatores externos que interferem no processo de absorção de água pelo gel. ${ }^{4}$

Hidrogéis à base de argilominerais, tais como, caulim, ${ }^{5}$ montmorilonita, ${ }^{6}$ atapulgita, ${ }^{7}$ vermiculita,${ }^{8}$ dentre inúmeros outros, têm despertado grande interesse por apresentarem vantagens como o baixo custo e a melhoria das propriedades dos hidrogéis compósitos como, por exemplo, aumento da estabilidade térmica, da capacidade de absorção e da velocidade de absorção de água. ${ }^{9}$

O caulim é o nome comercial dado a um tipo de argila, de coloração branca, composta principalmente pelo mineral caulinita. Os caulins são aluminossilicatos hidratados, cuja composição química aproxima-se de $\mathrm{Al}_{2} \mathrm{Si}_{2} \mathrm{O}_{5}(\mathrm{OH})_{4}$, o que corresponde a cerca de $46 \%$

*e-mail: almeida_quimica@yahoo.com.br de $\mathrm{SiO}_{2}, 40 \%$ de $\mathrm{Al}_{2} \mathrm{O}_{3}$ e $14 \%$ de $\mathrm{H}_{2} \mathrm{O}$ (em massa). Há também, no caulim, a presença de outros elementos, em menores quantidades, tais como, ferro, titânio, manganês, magnésio, potássio e sódio. ${ }^{10} \mathrm{~A}$ caulinita é o argilomineral mais frequentemente encontrado na natureza. A estrutura da caulinita é formada pelo empilhamento regular de camadas $1: 1$, isto é, cada camada é constituída de uma folha de sílica tetraédrica (quatro átomos de oxigênio ligados a um átomo de silício) e uma folha de alumina octaédrica (oito átomos de oxigênio ligados a um átomo de alumínio), ${ }^{11,12}$ também chamada folha de gibsita, ligadas entre si em uma única camada, através de um átomo de oxigênio em comum, dando uma estrutura fortemente polar. ${ }^{10}$ As ligações entre as camadas são feitas por ligações de hidrogênio, $\mathrm{OH}$ $\mathrm{O}$, uma vez que existe um plano de íons hidroxila numa das faces da camada e um plano de íons oxigênio na outra camada adjacente. ${ }^{11,12}$

O presente trabalho teve como objetivo avaliar o efeito da constituição dos caulins do nordeste brasileiro nas propriedades físico-químicas de hidrogéis compósitos de copolímero acrilamida-acrilato, com interesse no estudo desses sistemas para aplicação como condicionadores de solo. Para a caracterização dos hidrogéis foram utilizadas as técnicas de FTIR e DRX. Foram investigadas as capacidades de intumescimento dos hidrogéis, contendo diferentes quantidades de caulinita e diferentes graus de reticulação. Hidrogéis análogos, não contendo caulinita, foram sintetizados e utilizados como controle.

\section{PARTE EXPERIMENTAL}

\section{Materiais}

Os monômeros acrilamida (AAm), ácido acrílico (AAc), o acelerador da reação N,N,N',N'-tetrametilenodiamino (TEMED), o reticulante N,N'-metilenobisacrilamida (MBAAm) e o iniciador persulfato de potássio $\left(\mathrm{K}_{2} \mathrm{~S}_{2} \mathrm{O}_{8}\right)$ foram todos adquiridos da Sigma Aldrich. $\mathrm{O}$ sal de acrilato foi obtido por meio da neutralização do 
ácido acrílico com hidróxido de potássio (KAAc).

Dois caulins foram objetos deste estudo, ambos provenientes de estados do nordeste do Brasil, sendo um branco (CAULB) proveniente da Paraíba e o outro cinza (CAULC), proveniente do Piauí. Estes materiais foram secos em estufa a aproximadamente $90{ }^{\circ} \mathrm{C}$ por $24 \mathrm{~h}$, depois foram macerados e peneirados (peneira de malha 200 mesh, $<74 \mu \mathrm{m})$ e, em seguida, foram armazenados em dessecador.

\section{Síntese do hidrogel de copolímero de acrilamida-acrilato (PAMACRIL)}

A $30 \mathrm{~mL}$ de água destilada, sob agitação e borbulhamento de gás nitrogênio (para diminuir o efeito de inibição do $\mathrm{O}_{2}$ na reação de polimerização radicalar) foram adicionados $2,10 \mathrm{~g}$ de acrilamida e 3,30 g de acrilato. Após $10 \mathrm{~min}$ foram adicionados 16,2 $\mathrm{mg}$ de $\mathrm{K}_{2} \mathrm{~S}_{2} \mathrm{O}_{8}$. Em seguida, foi adicionado MBAAm $(0,05 ; 0,10 ; 0,15$ e 0,20 mol\% em relação à quantidade total de monômeros (AAm + KAAc) e $100 \mathrm{~mL}$ de solução de TEMED $0,57 \mathrm{~g} / \mathrm{L}$. O sistema foi fechado e mantido sob agitação e fluxo de $\mathrm{N}_{2}$ até a formação do gel. Após, o sistema reacional foi deixado em repouso por $15 \mathrm{~h}$. O material obtido, intumescido, foi dividido em pequenos pedaços e lavado com água destilada para se retirar o excesso de monômeros que não reagiram. O material foi seco em estufa a uma temperatura de $70{ }^{\circ} \mathrm{C}$ e depois macerado até granulometria de 9-24 mesh.

\section{Sínteses dos hidrogéis compósitos de copolímero de acrilamida- acrilato e caulim (CAULBG e CAULCG)}

As sínteses do CAULBG (hidrogel compósito de copolímero de acrilamida-acrilato e caulim branco) e do CAULCG (hidrogel compósito de copolímero de acrilamida-acrilato e caulim cinza) foram feitas da mesma forma que para a síntese do PAMACRIL, com a diferença que os monômeros foram dissolvidos em uma dispersão dos minerais $(1,5,10$ e $20 \% \mathrm{~m} / \mathrm{m}$ em relação aos monômeros).

\section{Métodos de caracterização}

\section{Análise química}

As amostras de caulins utilizadas nas análises químicas foram abertas usando-se o método de fusão, para análise do teor de Si e Al, e o método de fluorização, para análise do teor dos demais constituintes. As análises químicas foram realizadas usando espectrômetro de absorção atômica, modelo Spectra AA 110, Varian. ${ }^{13}$

\section{Umidade e perda ao fogo}

Dois cadinhos de porcelana foram calcinados em mufla a $850{ }^{\circ} \mathrm{C}$ por $30 \mathrm{~min}$ e pesados após esfriados. Cerca de $100 \mathrm{mg}$ dos caulins foram adicionados a cada cadinho e levados a estufa por $1 \mathrm{~h}$ a 100 ${ }^{\circ} \mathrm{C}$. Em seguida, foram retirados e esfriados em dessecador e depois pesados. Posteriormente, o mesmo material foi levado à mufla, aquecido por $30 \mathrm{~min}$ a $850^{\circ} \mathrm{C}$ e, depois de esfriado em dessecador, foi pesado novamente até massa constante.

\section{Espectroscopia na região do infravermelho}

Os espectros FTIR dos caulins e dos hidrogéis sintetizados foram obtidos em equipamento Shimadzu FT IR-8500, operando na faixa de $400-4000 \mathrm{~cm}^{-1}$. Para isso, as amostras foram preparadas em pastilha de $\mathrm{KBr}$ a $1 \%$ m-m.

\section{Difração de Raios- $X$}

Os difratogramas de Raios- $X$ foram obtidos através do difratômetro Rigaku (DMAXB), raios-X de pó, com radiação $\mathrm{Cu}-\mathrm{K}$ variando 2 de 3 a $30^{\circ}$.

\section{Estudos das propriedades}

\section{Experimentos de intumescimento}

Os hidrogéis obtidos foram caracterizados quanto à capacidade de absorção de água, através de ensaios de intumescimento. Para isso, cerca de $15 \mathrm{mg}$ de cada um dos géis foram colocadas em diferentes cadinhos filtrantes de $30 \mathrm{~mL}$ (porosidade $\mathrm{n}^{\circ} 0$ ), umedecidos previamente e com suas paredes externas secas. Cada conjunto foi inserido em água, de modo que o gel ficasse totalmente submerso.

O conjunto cadinho/gel foi retirado em vários intervalos de tempo, a parede externa do cadinho foi seca e o sistema foi pesado. Cada experimento foi realizado em triplicata. Os experimentos foram monitorados a partir da Equação 1:

$$
W=\left[m / m_{0}\right]-1
$$

onde $\mathrm{W}$ é o ganho de massa de água por grama de gel, $\mathrm{m}$ é a massa do material intumescido e $\mathrm{m}_{0}$ é a massa do material seco. ${ }^{14}$ Foi possível acompanhar a cinética de intumescimento no meio estudado plotando-se W como função do tempo de imersão.

\section{$O$ efeito da força iônica e do $\mathrm{pH}$}

Os hidrogéis foram imersos em diferentes soluções salinas $(\mathrm{NaCl}$, $\mathrm{NaHCO}_{3}, \mathrm{CaCl}_{2}$ ) com concentração de $0,15 \mathrm{~mol} \mathrm{~L}^{-1}$. O efeito do $\mathrm{pH}$ do meio de intumescimento também foi avaliado utilizando-se solução de $\mathrm{pH}$ 2,4 e soluções tampão de pH 9,4 e 7,4 (tampão fosfato), mantendo a força iônica constante $\left(\mathrm{I}=0,1 \mathrm{~mol} \mathrm{~L}^{-1}\right)$. A absorção de água foi determinada de acordo com o método descrito anteriormente. ${ }^{14}$

\section{RESULTADOS E DISCUSSÃO}

\section{Caracterização}

As análises químicas permitiram obter a composição química das argilas, sob a forma de seus óxidos. A razão molar silício/alumínio pode ser obtida através da fórmula mínima $\left(\mathrm{Si}_{2} \mathrm{Al}_{2} \mathrm{O}_{5}(\mathrm{OH})_{4}\right)$ da caulinita e possui valor 1. Estequiometricamente pode-se obter, a partir dos valores observados na Tabela 1, uma razão Si/Al experimental de 1,06 em termos de porcentagem de massa para a caulinita presente no caulim branco, sendo esses valores indicativos da presença de caulinita no caulim branco.

Tabela 1. Porcentagens (\%) das espécies químicas e de perda ao fogo presentes nos caulins precursores

\begin{tabular}{ccc}
\hline \multirow{2}{*}{ Espécie } & \multicolumn{2}{c}{ Massa (\%) } \\
\cline { 2 - 3 } & CAULB & CAULC \\
\hline $\mathrm{Na}_{2} \mathrm{O}$ & 0,60 & 5,90 \\
$\mathrm{~K}_{2} \mathrm{O}$ & 0,05 & 17,70 \\
$\mathrm{MgO}$ & 0,04 & 0,20 \\
$\mathrm{CaO}$ & Traços & 0,08 \\
$\mathrm{Fe}_{2} \mathrm{O}_{3}$ & 0,60 & 1,17 \\
$\mathrm{SiO}_{2}$ & 52,84 & 54,00 \\
$\mathrm{Al}_{2} \mathrm{O}_{3}$ & 44,00 & 14,30 \\
Perda ao fogo & 1,87 & 6,65 \\
\hline
\end{tabular}

Os valores de perda de massa ao fogo indicam que há presença de matéria orgânica em ambos os caulins, mas foi verificado que o caulim cinza (CAULC) possui quase o triplo de perda ao fogo que o caulim branco (CAULB). ${ }^{15}$ Tal perda está associada à grande quantidade de 
água de hidratação retida na argila CAULC. A elevada concentração de potássio evidenciada para o caulim cinza é um indicativo da presença de mica. Em consequência desse fato, a concentração de alumínio tende a ser menor que a esperada para a forma pura do caulim.

Na Figura 1i são mostrados os espectros de FTIR dos caulins e dos hidrogéis sintetizados. Os caulins contêm dois grupos de hidroxilas: as externas, também chamadas de hidroxilas de superfície interna, e as internas. As absorções em 3695 e $3650 \mathrm{~cm}^{-1}$ são referentes às hidroxilas externas e em 3622 e $914 \mathrm{~cm}^{-1}$, às hidroxilas octaédricas internas. ${ }^{15,16} \mathrm{~A}$ banda em $3440 \mathrm{~cm}^{-1}$ está relacionada com ligações de hidrogênio. $\mathrm{O}$ caulim tem bandas bem definidas na região que vai de $1400-400 \mathrm{~cm}^{-1}$, devido aos modos vibracionais característicos. ${ }^{17-19} \mathrm{As}$ bandas intensas registradas em 1115, 1036 e $1012 \mathrm{~cm}^{-1}$ são atribuídas aos estiramentos de Si-O-Si. As bandas em 789 e $749 \mathrm{~cm}^{-1}$, de intensidades relativas aproximadamente iguais, caracterizam a existência de uma caulinita bem cristalizada e/ou pouco substituída. ${ }^{17-19}$ O espectro FTIR do PAMACRIL (curva c da Figura 1i) caracteriza a formação do copolímero, devido ao aparecimento da banda em $1670 \mathrm{~cm}^{-1}$, referente ao estiramento $\mathrm{C}=\mathrm{O}$, e da banda em $1564 \mathrm{~cm}^{-1}$, referente ao estiramento $\mathrm{N}-\mathrm{H}$. Os espectros da CAULBG10 (curva d da Figura 1i) e CAULCG10 (curva e da Figura 1i) confirmam a presença da caulinita nos compósitos, devido ao aparecimento das bandas em 1115, 1036, 916, 543 e $464 \mathrm{~cm}^{-1} .^{17-19}$
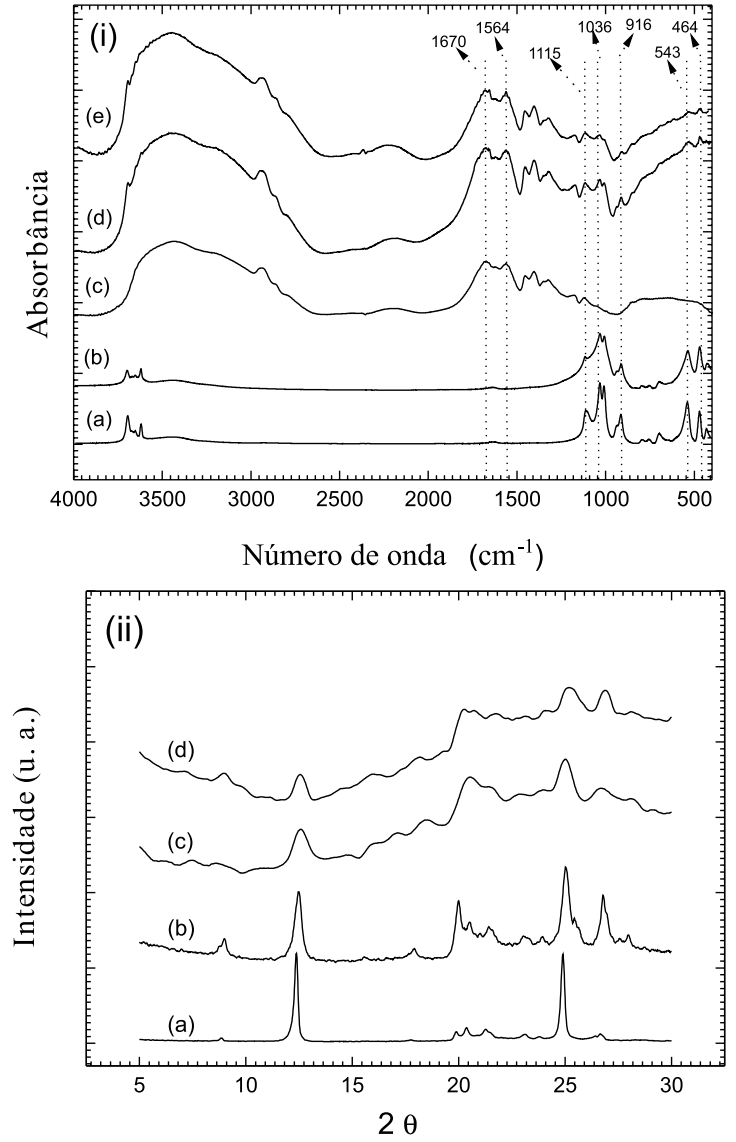

Figura 1. (i) Espectros de infravermelho do caulim branco (a), caulim cinza (b), PAMACRIL (c), CAULBG10 (d) e CAULCG10 (e). (ii) Difratogramas de Raios-X de CAULB (a), CAULC (b), CAULBG10 (c) e CAULCG10 (d)

Nos difratogramas de raios- $\mathrm{X}$ dos caulins (curvas a e b da Figura 1ii), observam-se os picos d(001), d(020) e d(002), referentes à caulinita, e aparecem em 2q igual a 12,41; 20,39 e 24,96 e que são, respectivamente, equivalentes às seguintes distâncias interplanares: 7,18; 4,46 e 3,58 A..$^{20-22}$ A identificação das fases da amostra indica, ainda, a presença de traços de quartzo $\left(21,30^{\circ}\right)$ para o CAULB e traços de mica para o CAULC, podendo haver interestratificação entre os dois minerais, causando coincidência de picos..$^{20-22}$ Pode-se observar também que o CAULC é menos cristalino que o CAULB. Tal fato pode ser atribuído ao maior teor de matéria orgânica no CAULC, como observado anteriormente pelos resultados da análise química.

Os difratogramas de CAULBG10 e CAULCG10 (curvas c e d da Figura 1ii) mostram um aumento da parte amorfa do material em relação aos difratogramas dos caulins, devido à formação do copolímero. No entanto, nos difratogramas dos compósitos CAULBG10 e CAULCG10 há a presença dos picos característicos da caulinita $(2 \theta \cong$ 12,20 e $\left.25^{\circ}\right)$ e quartzo $\left(2 \theta \cong 21^{\circ}\right)$ mostrando que as estruturas cristalinas dos caulins não foram totalmente destruídas e que houve a formação de compósitos, como observado anteriormente através dos espectros de FTIR. Os resultados observados nos difratogramas de raios-X e corroborados pelos espectros de FTIR confirmam a formação do hidrogel compósito e sugerem a presença dos caulins na matriz polimérica.

\section{Cinéticas de intumescimento}

Hidrogéis macromoleculares geralmente são constituídos de componentes hidrofílicos e/ou hidrofóbicos e de agente reticulante. $\mathrm{O}$ processo de intumescimento de um hidrogel é governado por forças físicas e químicas que induzem a entrada de líquido na matriz, sendo que o equilíbrio de intumescimento é atingido quando estas forças são balanceadas pela resposta elástica das cadeias constituintes de sua matriz, que é uma força que se opõe ao intumescimento. ${ }^{23} \mathrm{O}$ intumescimento deve-se à penetração de água na matriz polimérica por capilaridade e difusão. Dessa maneira, a água é absorvida por grupos hidrofílicos como hidroxilas, carboxilatos e aminos através da formação de ligação de hidrogênio. ${ }^{24} \mathrm{O}$ processo de intumescimento dos hidrogéis pode ser controlado pela variação de sua composição, pela quantidade de reticulação e por fatores externos, como a presença de sais, $\mathrm{pH}$ do meio e temperatura, fatores que são de fundamental importância no mecanismo de absorção de água por um hidrogel. ${ }^{23}$

A Figura 2a mostra a cinética de intumescimento em água dos hidrogéis compósitos (CAULBG10 e CAULCG10) e do hidrogel na ausência do caulim (PAMACRIL). Verifica-se um rápido crescimento no grau de intumescimento nos primeiros 30 min de imersão, atingindo cerca de $90 \%$ do valor de equilíbrio nesse intervalo de tempo, seguido de um processo mais lento até alcançar o equilíbrio $\left(\mathrm{W}_{\mathrm{eq}}\right)$, que ocorre por volta dos $60 \mathrm{~min}$, cujo valor do grau de intumescimento depende da constituição do hidrogel. Observou-se que os géis sintetizados apresentaram uma capacidade de absorção no equilíbrio $\left(\mathrm{W}_{\mathrm{eq}}\right)$ de $645 \pm 21,1016 \pm 23$ e $889 \pm 14 \mathrm{~g} / \mathrm{g}$, respectivamente, para PAMACRIL, CAULBG10 e CAULCG10, mostrando assim que os hidrogéis que possuem argila apresentam maior capacidade de absorção de água. Podem ocorrer possíveis reações entre grupos - $\mathrm{COO}^{-} \mathrm{e}$ os grupos $-\mathrm{OH}^{-}$na superfície dos caulins. ${ }^{25}$ Isso proporciona um aumento nas interações da superfície do gel com a água, acarretando uma maior absorção.

Para melhor avaliar o processo de intumescimento e o efeito da incorporação dos caulins sobre a cinética de intumescimento dos hidrogéis compósitos, foram empregadas as relações utilizadas por Karadag et al.. ${ }^{26}$ Algumas das características extraídas das curvas de intumescimento são:

onde

$$
t / W=A+B t
$$

$$
A=\frac{1}{k_{s} W_{t}^{2}}
$$

e

$$
B=\frac{1}{W_{t}}
$$



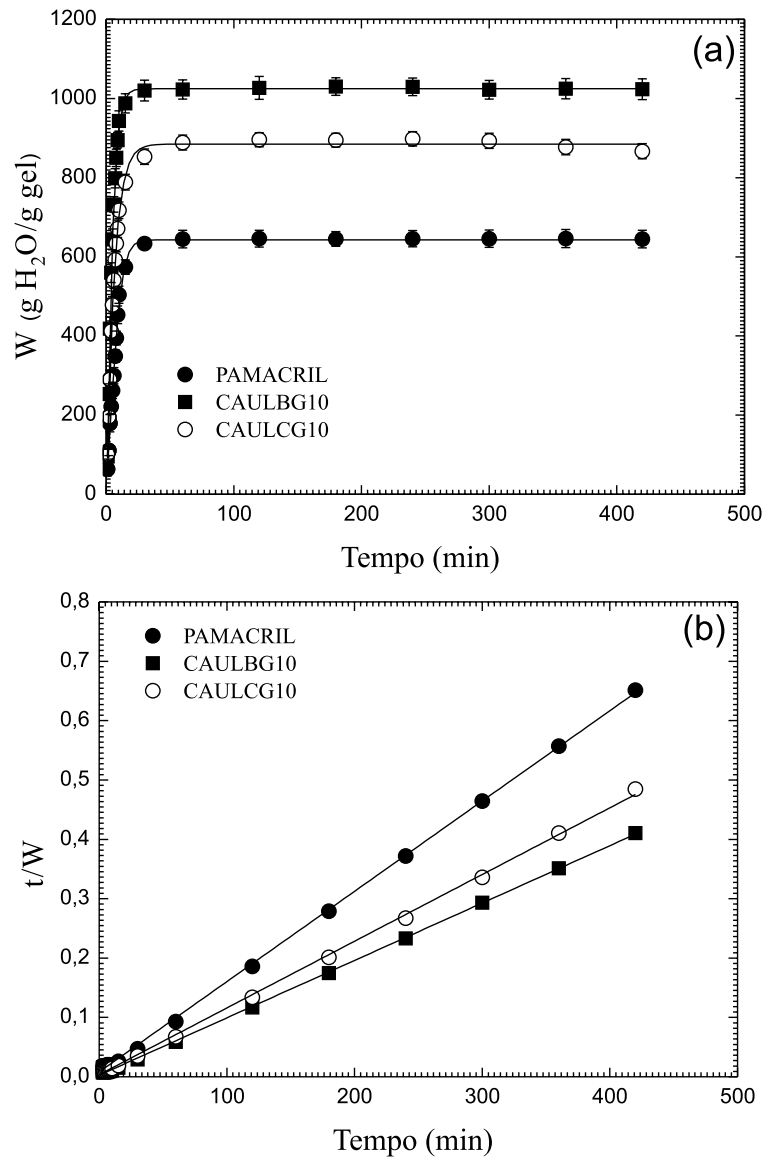

Figura 2. (a) Intumescimento em função do tempo de imersão e (b) gráfico de $t / W$ em função do tempo de imersão para os géis sintetizados

$\mathrm{O}$ parâmetro $A$ corresponde à taxa de intumescimento inicial $\left[(\mathrm{dW} / \mathrm{dt})_{0}\right]$ do hidrogel, $k_{s}$ é a constante de intumescimento e $W$ é o valor do intumescimento teórico no equilíbrio. Aplicando as Equações 2, 3 e 4 aos dados da cinética de intumescimento dos hidrogéis compósitos (Figura 2a), obtiveram-se os ajustes das curvas, mostradas na Figura $2 b$, a partir da qual se obtiveram os valores de $k_{s}$ e $W_{t}$ mostrados na Tabela 2 .

Tabela 2. Parâmetros obtidos na cinética de intumescimento

\begin{tabular}{lcccc}
\hline Hidrogel & ${ }^{\mathrm{a}} \mathrm{W}_{\mathrm{eq}}$ & ${ }^{\mathrm{b}} \mathrm{W}_{\mathrm{t}}$ & ${ }^{\mathrm{c}} \mathrm{t}_{\mathrm{eq}}(\mathrm{min})$ & ${ }^{\mathrm{d}} \mathrm{k}_{\mathrm{s}}\left(\mathrm{min}^{-1}\right)$ \\
\hline PAMACRIL & $645 \pm 21$ & 658 & $24 \pm 3$ & $6.54 \times 10^{-4}$ \\
CAULBG10 & $1016 \pm 23$ & 1031 & $18 \pm 1$ & $3.12 \times 10^{-4}$ \\
CAULCG10 & $889 \pm 14$ & 893 & $21 \pm 2$ & $3.52 \times 10^{-4}$ \\
\hline
\end{tabular}

Intumescimento no equilíbrio ( $\mathrm{g} \mathrm{H}_{2} \mathrm{O} / \mathrm{g}$ gel); ${ }^{\mathrm{b} I n t u m e s c i m e n t o ~ t e o ́ r i c o ~ n o ~}$ equilíbrio ( $\mathrm{g} \mathrm{H}_{2} \mathrm{O} / \mathrm{g}$ gel); ${ }^{\mathrm{c}}$ Tempo de equilíbrio (min); ${ }^{\mathrm{d}}$ Taxa de intumescimento $\left(\mathrm{g} \mathrm{gel} / \mathrm{gH}_{2} \mathrm{O}\right) / \mathrm{min}$

\section{Efeito da quantidade de caulim nos géis}

A relação entre absorção de água e quantidade dos caulins presentes nos hidrogéis compósitos é mostrada na Figura 3. A presença de caulim na matriz do hidrogel aumenta sua capacidade de intumescimento em relação ao PAMACRIL, cujo valor do incremento é 58 e 39\%, respectivamente, para CAULBG10 e CAULCG5. De acordo com Xie e Wang, ${ }^{25}$ cátions presentes na argila aumentam a hidrofilicidade do compósito e a diferença de pressão osmótica entre o polímero e a solução externa. Pode-se ver que a incorporação de 5 e $10 \%$ de argila nos hidrogéis possibilita a ocorrência de um máximo de absorção de água para os sistemas CAULCG5 e CAULBG10, respectivamente. No entanto, um subsequente aumento dos percentuais de argila nos compósitos resultou em uma significante redução do intumescimento. O decréscimo em $\mathrm{W}_{\mathrm{eq}}$, observado quando quantidades maiores de caulim foram utilizadas na formulação do hidrogel, pode ser atribuído à formação de pontos adicionais de reticulação na rede polimérica devido à presença da argila na matriz, proporcionando um aumento na densidade de reticulação no compósito e, portanto, a diminuição da elasticidade do polímero. ${ }^{27,28}$

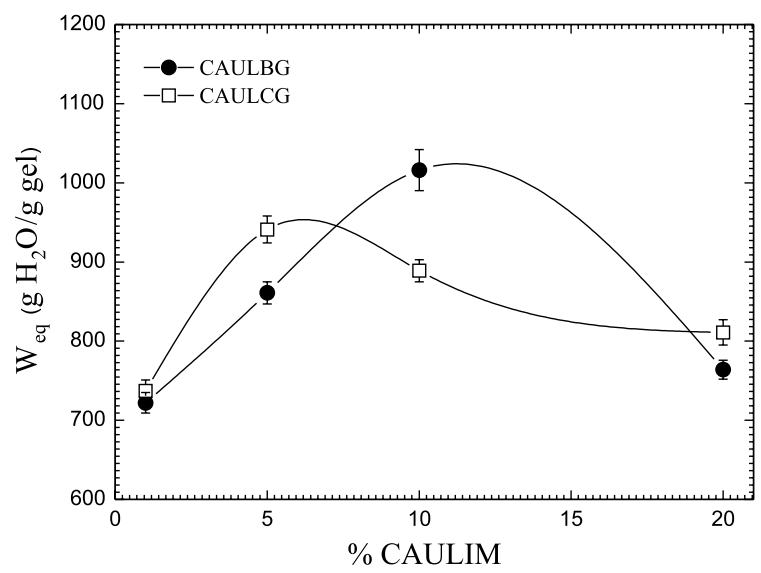

Figura 3. Efeito da quantidade de argila no processo de intumescimento

\section{Efeito do grau de reticulação}

Foram sintetizados hidrogéis usando diferentes concentrações do agente de reticulação MBAAm, mantendo-se as concentrações de acrilamida e de acrilato de potássio em 50\%. A faixa de concentração de MBAAm estudada foi de 0,05-0,20 mol\%. De acordo com a teoria de Flory, ${ }^{23}$ a densidade de reticulação é um fator chave que influencia a capacidade de absorção de água, que é inversamente proporcional à densidade de ligações cruzadas. A dependência da quantidade de água absorvida no equilíbrio $\left(\mathrm{W}_{\mathrm{eq}}\right)$ na concentração MBAAm é mostrada na Figura 4, na qual se pode observar claramente que o aumento da concentração do agente de reticulação MBAAm provocou um pronunciado decréscimo na absorção de água dos hidrogéis (de $1184 \pm$ 19 para $875 \pm 20 \mathrm{~g} \mathrm{H}_{2} \mathrm{O} / \mathrm{g}$ gel e de $1043 \pm 20$ para $794 \pm 16 \mathrm{~g} \mathrm{H}_{2} \mathrm{O} / \mathrm{g}$ gel, respectivamente, para CAULBG e CAULCG). De maneira geral, redes poliméricas formadas com alta concentração de MBAAm são mais fortemente reticuladas e isso reflete em uma menor expansão e menor difusão de volume de água para o interior da matriz. ${ }^{29-31}$

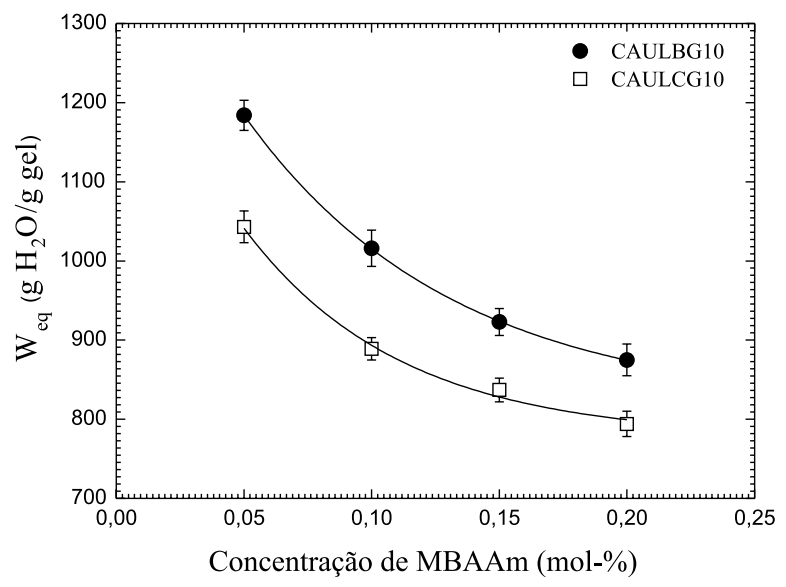

Figura 4. Efeito da concentração de reticulante na cinética de intumescimento em água para os hidrogéis compósitos 


\section{Efeito do pH e da força iônica}

$\mathrm{O}$ efeito do $\mathrm{pH}$ no processo de intumescimento de hidrogéis é de grande importância. Com a variação do $\mathrm{pH}$ do meio frequentemente ocorre uma flutuação no volume livre accessível para a penetração de moléculas de água, o que afeta as características de intumescimento do hidrogel..$^{32}$

O efeito do $\mathrm{pH}$ na cinética de intumescimento do hidrogel foi verificado com a utilização de soluções tampão com $\mathrm{pH}=2,5 ; 7,4$ e 9,4 mantendo força iônica constante $\left(I=0,1 \mathrm{~mol} \mathrm{~L}^{-1}\right)$. Os resultados são apresentados na Figura 5a. Observa-se aumento do valor de $\mathrm{W}_{\mathrm{eq}} \mathrm{com}$ o aumento do $\mathrm{pH}$ da solução. Em meio ácido os ânions carboxilato são protonados e as forças de repulsão ânion-ânion diminuem, o que conduz a um mínimo de intumescimento do hidrogel. Para valores de $\mathrm{pH}$ maiores, os grupos carboxilato são ionizados e a repulsão eletrostática entre os grupos $\mathrm{CCO}^{-}$causa o aumento de $\mathrm{W}_{\mathrm{eq}}{ }^{33}$
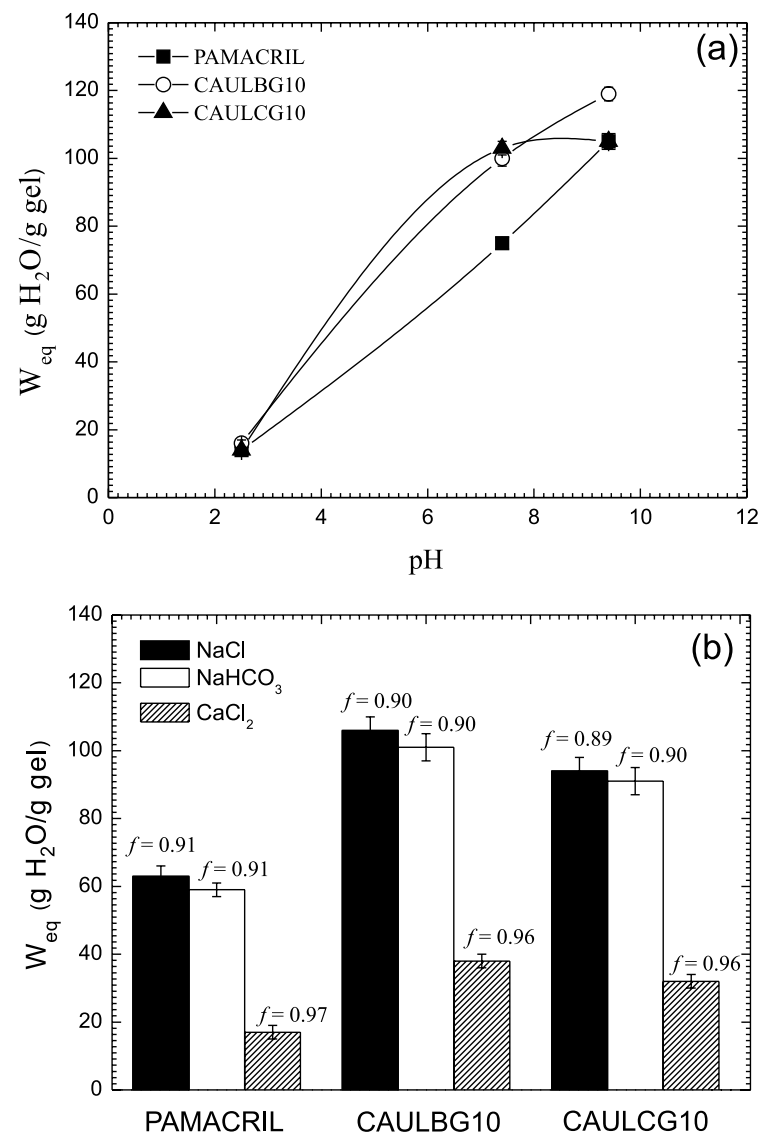

Figura 5. (a) Efeito do pH no intumescimento dos hidrogéis, (b) efeito da força iônica no intumescimento dos hidrogéis

Em pH 9,4 o hidrogel intumesce devido à repulsão dos grupos $\mathrm{COO}^{-}$, enquanto em $\mathrm{pH}$ 2,5 há o colapso do hidrogel dentro de poucos minutos, em virtude do efeito de blindagem do excesso de cátions. Esse comportamento em diferentes $\mathrm{pHs}$ torna esse sistema um forte candidato a ser utilizado em sistemas de liberação controlada de fertilizantes.

A presença de íons tem profundo efeito no comportamento de intumescimento de hidrogéis. O balanço entre a pressão osmótica do sistema e a resposta elástica do gel é um dos fatores que controlam a capacidade de absorção de água. A pressão osmótica resulta da diferença entre as concentrações de íons no interior do gel e na solução externa. ${ }^{34}$

$\mathrm{O}$ efeito da presença de íons nos hidrogéis foi investigado pela adição de $\mathrm{NaCl}, \mathrm{NaHCO}_{3}$ ou de $\mathrm{CaCl}_{2}$, com concentração fixada de $0,10 \mathrm{~mol} \mathrm{~L}^{-1}$. A Figura $5 \mathrm{~b}$ apresenta os resultados de intumescimento para os hidrogéis em solução de $\mathrm{NaCl}, \mathrm{NaHCO}_{3} \mathrm{e} \mathrm{CaCl}_{2}$. A sensibilidade de um material absorvente, por exemplo, um hidrogel, à presença de sais pode ser relacionada pelo fator adimensional $f$ definido como: ${ }^{35}$

$$
f=1-\left(\frac{W_{\text {salina }}}{W_{\text {agua }}}\right)
$$

onde $\mathrm{W}_{\text {salina }}$ e $\mathrm{W}_{\text {agua }}$ são os graus de intumescimento em equilíbrio em meio salino e em água pura, respectivamente.

Os valores de $f$ indicam que os géis na presença das argilas (CAULBG10 e CAULCG10) são um pouco menos sensíveis à presença de sais que o gel PAMACRIL. O aumento da força iônica reduz a diferença de concentração de íons móveis entre o polímero e a solução externa (pressão de intumescimento osmótico) e, de imediato, o volume de gel diminui, resultando no colapso do gel.

Quantidades de metais di e trivalentes podem diminuir drasticamente a capacidade de intumescimento dos hidrogéis, devido à habilidade de complexação dos grupos carboxamida ou carboxilato e consequente formação de complexos inter e intramolecular. ${ }^{36}$

\section{CONCLUSÕES}

Obteve-se um compósito constituído de poli(acrilamida-coacrilato) e dos diferentes caulins (CAULB ou CAULG), como verificado por FTIR e Raio-X. Os géis sintetizados mostraram-se mais eficientes na absorção de água do que o gel preparado sem o uso dos caulins. A caulinita presente na matriz copolimérica melhorou suas propriedades de absorção do material compósito em relação ao hidrogel de controle (PAMACRIL), proporcionando um aumento de 57 e $46 \%$ no valor do $\mathrm{W}_{\text {eq }}$, respectivamente, para CAULBG10 e CAULCG5, com 0,10 mol\% de MBAAm. A capacidade de absorção de água pelo hidrogel compósito depende da concentração e da constituição da argila e da concentração do agente de reticulação. Estes resultados, mesmo que preliminares, indicam que os géis preparados possuem grande potencial para serem utilizados no ramo agronômico. Entretanto, algumas variáveis terão de ser otimizadas para tal aplicação.

\section{AGRADECIMENTOS}

Ao apoio financeiro da FUNCAP (BPI 0280-106/08 e PIL 139.01.00/09) e do CNPq (Proc. 507308/2010-7).

\section{REFERÊNCIAS}

1. Raju, M. P.; Raju, K. M.; J. Appl. Polym. Sci. 2001, 80, 2635.

2. Bucholz, F. L.; Pepas, N. A., eds.; Superabsorbent Polymers Science and Technology, ACS Symposium Series 573, ACS Press: Washington DC, 1994, p. 121.

3. Aslam, M.; Polymers as soil conditioners and seating agents, Pakistan Academy of Sciences: Islamabad, 1990.

4. Rudzinski, W. E.; Dave, A. M.; Vaishnav, U. H.; Kumbar, S. G.; Kulkarni, A. R.; Aminabhavi, T. M.; Des. Monomers Polym. 2002, 5, 39.

5. Wu, J. H.; Wei, Y. L.; Lin, J.; Lin, S.; Polymer 2003, 44, 6513.

6. Janovák, L.; Varga, J.; Kemény, L.; Dékány, I.; Appl. Clay Sci. 2009, 43, 260.

7. Wang, W.; Zheng, Y.; Wang, A.; Polym. Adv. Technol. 2008, 19, 1852.

8. Wang, Q.; Xie, X.; Zhang, X.; Zhang, J.; Wang, A.; Int. J. Biol. Macromol. 2010, 46, 356.

9. Haraguchi, K.; Takehisa, T.; Adv. Mater. 2002, 14, 1120.

10. Araújo, J. C.; Assis, J. T.; Monine, V. I.; Bertolino, L. C.; Rev. Mater. 2006, 11, 361 . 
11. Elbokl, T. A.; Detellie, C.; J. Phys. Chem. Solids 2006, 67, 950.

12. Hu, Y.; Liu, X.; Miner. Eng. 2003, 16, 1279.

13. Gardolinski, J. E.; Martins, H. P.; Wypych, F.; Quim. Nova 2003, 26, 30.

14. Cândido, J. S.; Leitão, R. C. F.; Ricardo, N. M. P. S.; Feitosa, J. P. A.; Muniz, E. C.; Rodrigues, F. H. A.; J. Appl. Polym. Sci. 2012, 123, 879.

15. Gardolinski, J. E.; Wypych, F.; Quim. Nova 2001, 24, 761.

16. Frost, R. L.; Locos, O. B.; Kristof, J.; Kloprogge, J. T.; Vib. Spectrosc. 2001, 26, 33.

17. Fernandes, M. V. S.; Silva, L. R. D.; Cerâmica 2011, 57, 219.

18. Gardolinski, J. E.; Carrera, L. C. M.; Cantão, M. P.; Wypych, F.; J. Mater. Sci. 2000, 35, 3113.

19. Gardolinski, J. E.; Zamora, P. P.; Wypych, F.; J. Colloid Interface Sci. 1999, 211, 137.

20. Gardolinski, J. E.; Ramos, L. P.; Souza, G. P.; Wypych, F.; J. Colloid Interface Sci. 2000, 221, 284.

21. Tsunematsu, K.; Tateyama, H.; J. Am. Ceram. Soc. 1999, 82, 1589.

22. Silva, D. A.; Tese de Doutorado, Universidade Federal do Ceará, Brasil, 2006.

23. Flory, P. J. Em Principles of Polymer Chemistry; Ithaca; Cornell University Press: New York, 1953.
24. Zheng, Y.; Li, P.; Zhang, J.; Wang, A.; Eur. Polym. J. 2007, 43, 1691

25. Xie, Y.; Wang, A.; J. Polym. Res. 2009, 16, 143.

26. Karadag, E.; Uzum, O. B.; Saraydin, D.; Mater. Des. 2005, 26, 265.

27. Wu, J. H.; Wei, Y. L.; Lin, J.; Lin, S.; Polymer 2003, 44, 6513.

28. Li, A.; Wang, A.; Chen, J.; J. Appl. Polym. Sci. 2004, 92, 1596.

29. Spagnol, C.; Rodrigues, F. H. A.; Carvalho Neto, A. G. V.; Pereira, A. G. B.; Fajardo, A. R.; Radovanovic, E.; Rubira, A. F.; Muniz, E. C.; Eur. Polym. J. 2012, 48, 454.

30. Spagnol, C.; Rodrigues, F. H. A.; Pereira, A. G. B.; Fajardo, A. R.; Rubira, A. F.; Muniz, E. C.; Carbohydr. Polym. 2012, 87, 2038.

31. Spagnol, C.; Rodrigues, F. H. A.; Pereira, A. G. B.; Fajardo, A. R.; Rubira, A. F.; Muniz, E. C.; Cellulose 2012, 19, 1225.

32. Bajpai, A. K.; Giri, A.; React. Funct. Polym. 2002, 53, 125.

33. Hamshary, H. E.; Eur. Polym. J. 2007, 43, 4830.

34. Molloy, J. P.; Smith, M. J.; Cowling, M. J.; Mater. Des. 2000, 21, 169.

35. Pourjavadi, A.; Sadeghi, M.; Hoseinzadeh, H.; Polym. Adv. Technol. 2004, 15, 645 .

36. Mahdavinia, G. R.; Zohuriaan-Mehr, M. J.; Pourjavadi, A.; Polym. Adv. Technol. 2004, 15, 173. 